\title{
Discrete Differential Evolution for Mixed Discrete
}

\section{Non-Linear Problems}

\author{
Satoshi Kitayama ${ }^{1}$, Masao Arakawa ${ }^{2}$ and Koetsu Yamazaki ${ }^{1}$ \\ 1. College of Science and Engineering, Kanazawa University, Kanazawa 920-1192, Japan \\ 2. Kagawa University, Hayashi-cho, Takamatsu, Kagawa 761-0396, Japan
}

\begin{abstract}
Differential evolution (DE) is a global optimizer for continuous design variables. To enhance DE, it is necessary to handle discrete design variables. In this paper, a discrete differential evolution (DDE) algorithm is proposed to handle discrete design variables. The proposed DDE is based on the DE/1/rand/bin method. In the proposed DDE, the mutation ratio is regarded as the exchange probability, and thus, no modifications of $\mathrm{DE} / 1 / \mathrm{rand} /$ bin are required. In addition, in order to maintain diversity through the search process, we initialize all search points. By introducing the initialization of all search points, global or quasi-optimum solution can be found. We validate the proposed DDE by applying it to several benchmark problems.
\end{abstract}

Key words: Global optimization, differential evolution, mixed-discrete nonlinear problems.

\section{Introduction}

Population-based optimization techniques such as particle swarm optimization (PSO), genetic algorithm (GA), and ant colony optimization (ACO) are widely studied in the field of evolutionary computation. Differential evolution (DE), proposed by Storn and Price, is a population-based optimization technique [1] used to obtain a global minimum solution in continuous design variable problems [2-4]. DE, as well as other population-based optimization techniques, is also considered as a stochastic optimization technique. Several DE versions have been proposed thus far. The basic $\mathrm{DE}$ version is called $\mathrm{DE} / 1 / \mathrm{rand} / \mathrm{bin}$, and is explained in section 2. In $\mathrm{DE} / 1 / \mathrm{rand} / \mathrm{bin}$, numerous search points are randomly distributed throughout the design variable space, and three search points are randomly selected. In order to produce a new search point, mutation and crossover operations are applied. The objective function at the current iteration is compared with that for the new search point. If the

Corresponding author: Satoshi Kitayama, PhD, research fields: engineering optimization. E-mail: kitagon@t.kanazawa-u.ac.jp. objective function is improved, the new search point is replaced. Using this procedure, a global minimum can be found. The effects of setting the parameter in DE are well examined through numerical experiments [5-8]. DE is well suited for finding a global minimum solution in continuous design variable problems. However, extending DE to handle discrete design variables remains an unresolved issue.

The simplest way for DE to handle discrete design variable is by applying rounding-off and truncation techniques. Therefore, all discrete design variables are assumed to be continuous, and the above techniques are applied to obtain the discrete optimum. Such approaches are currently employed due to their simplicity [9]. Although these techniques are valid in handling discrete design variables, some problems may arise. Let us consider the cases shown in Fig. 1, which illustrates some of the potential problems [10].

In Fig. 1a, $\boldsymbol{x}_{L}$ denotes the local minimum of the continuous design variables. Points A and B represent the discrete points of the $i$-th discrete design variable. If rounding-off is applied to $\boldsymbol{x}_{\mathrm{L}}$, point $\mathrm{B}$ is selected. However, in this case, the objective function is not improved compared to that of point A. Therefore, in 
some cases, rounding-off worsens the objective function. Next, let us consider Fig. 1b, in which no discrete optimum can be found by rounding-off and truncation. It can be seen in Fig. 1 that rounding-off and truncation sometimes lead to the wrong results. A summary of several optimization methods available for discrete design variables is presented in Ref. [11].

For the mixed discrete non-linear problems (MDNLPs), we have proposed the use of a particle swarm optimization algorithm [12], in which the discrete design variables are transformed into continuous ones using a penalty function [13]. By introducing the penalty function, it is possible to treat all design variables as continuous. The augmented objective function, which consists of the objective and the penalty function, is constructed. The augmented objective function has many local minima at the discrete points. The optimum of the discrete design variables corresponds to the global minimum of the augmented objective function. In the penalty function approach, the key is to adjust the appropriate penalty coefficient of the penalty function. To date, there is no effective way available to determine an appropriate penalty coefficient.

Some applications of DE to discrete design variables have been reported [14-16]; however, they employ simple rounding-off and truncation techniques. Application of DE to the flow-shop scheduling problems has also been reported [15, 16], but some modifications are required. These algorithms are limited to the flow-shop scheduling problems. For simplicity and versatility, it is preferable to apply

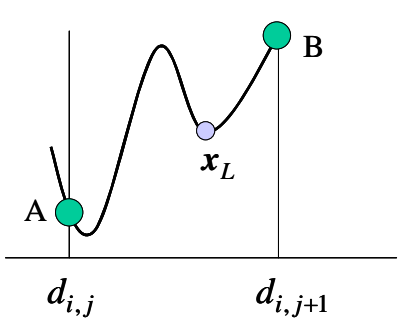

(a)
Optimum of continuous variables

(b)

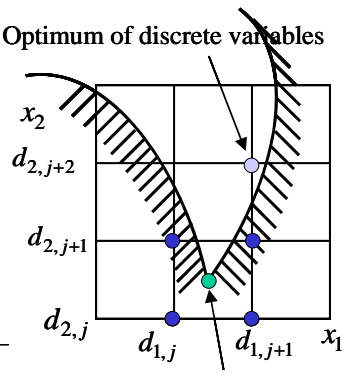

Fig. 1 Nature of the discrete optimization.
$\mathrm{DE} / 1 / \mathrm{rand} /$ bin without any modifications to the discrete design variable problems and MDNLPs. For example, PSO algorithms for the flow-shop scheduling problems have been reported in [17-19]. However, the applications of these algorithms are limited to the flow-shop scheduling problems. These algorithms cannot be applied to discrete design variables problems and MDNLPs.

In this paper, we propose a discrete differential evolution (DDE) algorithm to handle the discrete design variables. In the proposed DDE, the discrete design variables are handled directly. Therefore, rounding-off and truncation techniques are not employed in the proposed DDE. In the proposed DDE, the mutation ratio $F$ is regarded as the exchange probability, and no modifications of $\mathrm{DE} / 1 /$ rand/bin are required. Therefore, it is possible to directly handle the discrete design variables. In order to maintain diversity through the search process, we initialize all search points. Thus, when the standard deviation of objective function is less than a predefined threshold, all search points are randomly distributed in the design variable space. By initializing all search points, the global or quasi-optimum solution can be found.

The remainder of this paper is organized as follows: In section 2, DE for the continuous design variables is briefly explained. In section 3, the SDDE will be described and the algorithm will be shown. Through benchmark problems of the discrete design variable problems and the MDNLPs, the validity of the proposed DDE is examined.

\section{Differential Evolution}

\subsection{Problem Definition}

In general, MDNLP is described as follows [11]:

$$
\begin{gathered}
f(x) \rightarrow \min \\
x_{i}^{L} \leq x_{i} \leq x_{i}^{U} \quad i=1,2, \cdots, m \\
x_{m+i} \in Z_{i} \quad Z_{i}=\left\{z_{i, 1}, z_{i, 2}, \cdots, z_{i, q}\right\} \quad i=1,2, \cdots, n \\
g_{l}(x) \leq 0 \quad l=1,2, \cdots, \text { ncon }
\end{gathered}
$$

where $\boldsymbol{x}$ denotes the design variables, which consist of continuous and discrete design variables. $f(\boldsymbol{x})$ is the 
objective function to be minimized, and $g_{l}(x)$ is the behavior constraints. ncon represents the number of behavior constraints. $X_{i}$ represents the $i$-th design variable, and $m$ and $n$ are the total number of continuous and discrete design variables, respectively. $x_{i}^{L}$ and $x_{i}^{U}$ denote the lower and upper bounds of the continuous design variables, respectively. $Z_{i}$ is the set of discrete values for the $i$-th discrete design variable. $z_{i, j}$ is the $j$-th discrete value for the $i$-th discrete design variable. $q$ represents the number of discrete values. The lower and upper bounds on the discrete design variables are given by $z_{i, 1}$ and $z_{i, q}$, respectively. In general, the number of available discrete values $q$ for each discrete design variable may be different.

\subsection{Basic Algorithm of DE/1/Rand/Bin}

DE is a population-based optimization technique with no gradient information. Although several DE versions have been proposed, in this paper we focus on the basic DE version called DE/1/rand/bin. Here, the following descriptions are introduced:

$k$ : $k$-th iteration.

$\boldsymbol{x}_{i}^{k}$ : $i$-th search point at $k$-th iteration.

$d_{\text {max }}$ : number of search points.

$k_{\max }$ : number of search iterations.

$F$ : mutation ratio.

Cr: crossover ratio.

The $\mathrm{DE} / 1 /$ rand/bin algorithm is briefly summarized as follows:

(STEP1) $d_{\max }, k_{\max }, F$, and $C r$ are set. Initial search points are distributed at random. Iteration counter $k$ is set to 1 .

(STEP2) The following procedures are applied to all search points. $d$ is set to 1 .

(STEP2-1) For $\boldsymbol{x}_{d}{ }^{k}$, three search points $\left(\boldsymbol{x}_{r 1}{ }^{k}, \boldsymbol{x}_{r 2}{ }^{k}\right.$, $\left.\boldsymbol{x}_{r 3}{ }^{k}\right)$ are selected at random, where $d \neq r 1 \neq r 2 \neq r 3$. (STEP2-2) Mutation: a new point $\boldsymbol{v}_{d}{ }^{k}$ is produced by the following equation.

$$
\boldsymbol{v}_{d}^{k}=\boldsymbol{x}_{r 1}^{k}+F\left(\boldsymbol{x}_{r 2}^{k}-\boldsymbol{x}_{r 3}^{k}\right)
$$

(STEP2-3) Crossover: a new search point $\boldsymbol{u}_{d}{ }^{k}$ is produced by the crossover between $\boldsymbol{x}_{d}{ }^{k}$ and $\boldsymbol{v}_{d}{ }^{k}$.
(STEP2-4) The new search point $\boldsymbol{u}_{d}{ }^{k}$ is updated according to the following criterion:

$$
\left.\begin{array}{l}
f\left(\boldsymbol{u}_{d}^{k}\right) \leq f\left(\boldsymbol{x}_{d}^{k}\right) \rightarrow \boldsymbol{x}_{d}^{k}:=\boldsymbol{u}_{d}^{k} \\
f\left(\boldsymbol{u}_{d}^{k}\right)>f\left(\boldsymbol{x}_{d}^{k}\right) \rightarrow \boldsymbol{x}_{d}^{k}:=\boldsymbol{x}_{d}^{k}
\end{array}\right\}
$$

(STEP2-5) If $d$ is less than $d_{\max }, d$ is increased by 1 $(d=d+1)$. Return to STEP2-1.

(STEP3) Iteration counter is increased by $1(k=k+1)$.

(STEP4) If $k$ is less than $k_{\max }$, return to STEP2. Otherwise, the algorithm terminates.

From Eq. (6) it can be seen that the new point $\boldsymbol{u}_{d}{ }^{k}$ is replaced only when the objective function is improved. This enhances local search, while the random selection of the three search points facilitates a global search. Therefore, DE/1/rand/bin is considered to have the ability to search both locally and globally. An illustrative example how to produce the new search point $\boldsymbol{u}_{d}{ }^{k}$ is presented in Fig. 2.

First, the crossover point is randomly determined, and then the element of $\boldsymbol{u}_{d}{ }^{k}$ at the crossover point inherits from $\boldsymbol{v}_{d}^{k}$. In Fig. 2, the crossover point is 4 , and the element of $\boldsymbol{u}_{d}{ }^{k}$ inherits from the 4-th element of $\boldsymbol{v}_{d}{ }^{k}$. In order to determine the other elements of $\boldsymbol{u}_{d}{ }^{k}$, a random number $r$ between 0 and 1 is generated. If $r$ is less than $C r$, the element of $\boldsymbol{u}_{d}{ }^{k}$ inherits from $\boldsymbol{v}_{d}{ }^{k}$. Otherwise, the element of $\boldsymbol{u}_{d}{ }^{k}$ inherits from $\boldsymbol{x}_{d}{ }^{k}$.

\section{Proposed Discrete Differential Evolution}

In this section, DDE is described. The basic idea of the proposed DDE is obtained from the discrete particle swarm optimization (DPSO) [17-19]. In DPSO, the addition and subtraction of two vectors are defined. To move the search points on discrete points, the product of the coefficient and a vector is also defined. In $\mathrm{DE} / 1 / \mathrm{rand} / \mathrm{bin}$, due to the mutation it is not possible to move the search points on discrete points. It is clear from Eq. (5) that mutation consists of the following two terms:

$$
\begin{gathered}
\boldsymbol{D}^{k}=F\left(\boldsymbol{x}_{r 2}^{k}-\boldsymbol{x}_{r 3}^{k}\right) \\
\boldsymbol{v}_{d}^{k}=\boldsymbol{x}_{r 1}^{k}+F\left(\boldsymbol{x}_{r 2}^{k}-\boldsymbol{x}_{r 3}^{k}\right)=\boldsymbol{x}_{r 1}^{k}+\boldsymbol{D}^{k}
\end{gathered}
$$

By applying these two calculation rules in DPSO [20], the search points can move on discrete points. 


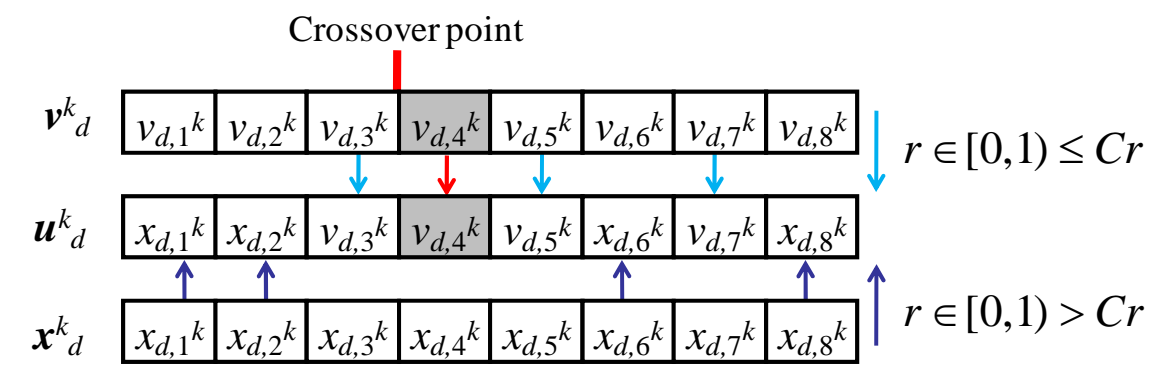

Fig. 2 An illustrative example of the crossover operation.

\subsection{Calculation Rules and Detailed Procedure}

In this subsection, we assume that all elements of $\boldsymbol{x}_{r 1}{ }^{k}, \boldsymbol{x}_{r 2}{ }^{k}$, and $\boldsymbol{x}_{r 3}{ }^{k}$ are discrete. It is important to move all search points on discrete points. In order to achieve this objective, the following two rules are employed.

RULE 1: Product of the coefficient and the difference vector [20]:

$$
\boldsymbol{x}=\alpha(\boldsymbol{y}-\mathbf{z})
$$

where

$$
x_{i}= \begin{cases}y_{i} & r \leq \alpha \\ z_{i} & r>\alpha\end{cases}
$$

In Eq. (9), $\boldsymbol{x}, \boldsymbol{y}$ and $\mathbf{z}$ represent the vectors, which contain discrete elements. $x_{i}, y_{i}$, and $z_{i}$ denote the $i$-th element of $\boldsymbol{x}, \boldsymbol{y}$, and $\mathbf{z}$. In Eq. (9), $\alpha$ is the coefficient, and in Eq. (10) $r$ is a random number between [0,1]. In order to determine the $i$-th element of $\boldsymbol{x}, \boldsymbol{r}$ is generated. If $r$ is less than $\alpha, x_{i}$ inherits from $y_{i}$. Otherwise, $x_{i}$ inherits from $z_{i}$. It is clear from Eq. (10) that $\alpha$ represents the exchange probability. Moreover, we can identify the similarities between Eqs. (7) and (9). In particular, $\alpha$ in Eq. (9) corresponds to $F$ in Eq. (7). Therefore, to determine $\boldsymbol{D}^{k}$, it is possible to regard the mutation ratio $F$ as the exchange probability. The elements of $\boldsymbol{D}^{k}$ can be determined as follows:

$$
D_{i}^{k}=\left\{\begin{array}{ll}
x_{x 3, i}^{k} & r \leq F \\
x_{r 2, i}^{k} & r>F
\end{array} i=1,2, \cdots, n\right.
$$

where $r$ represents a random number between $[0,1]$.

RULE 2: Addition of two vectors:

$$
\boldsymbol{a}=\boldsymbol{b}+\boldsymbol{c}
$$

where

$$
a_{i}= \begin{cases}c_{i} & r \leq 0.5 \\ b_{i} & r>0.5\end{cases}
$$

In Eq. (12), $\boldsymbol{a}, \boldsymbol{b}$ and $\boldsymbol{c}$ represent the vector, and all elements are discrete. In Eq. (13), $a_{i}, b_{i}$, and $c_{i}$ denote the $i$-th elements of $\boldsymbol{a}, \boldsymbol{b}$, and $\boldsymbol{c}$, respectively, and $r$ is a random number between $[0,1]$. In order to determine the $i$-th element of $\boldsymbol{a}, r$ is generated. If $r$ is less than 0.5, $a_{i}$ inherits from $c_{i}$. Otherwise, $a_{i}$ inherits from $b_{i}$. In addition, we can identify the similarities between Eqs. (8) and (12). Therefore, the following correspondence can be considered.

$$
\left.\begin{array}{rl}
\boldsymbol{v}_{d}^{k} & \leftrightarrow \boldsymbol{a} \\
\boldsymbol{x}_{r 1}^{k} & \leftrightarrow \boldsymbol{b} \\
\boldsymbol{D}^{k} & \leftrightarrow \boldsymbol{c}
\end{array}\right\}
$$

Hence, all elements of $\boldsymbol{v}_{d}{ }^{k}$ are stochastically determined by $\boldsymbol{x}_{r 1}{ }^{k}$ and $\boldsymbol{D}^{k}$.

The detailed procedure of mutation for discrete design variables is summarized as follows:

(M-STEP1) For $\boldsymbol{x}_{d}{ }^{k}$, three search points $\left(\boldsymbol{x}_{r 1}{ }^{k}, \boldsymbol{x}_{r 2}{ }^{k}\right.$, $\left.\boldsymbol{x}_{\mathrm{r} 3}{ }^{k}\right)$ are selected at random, where $d \neq r 1 \neq r 2 \neq r 3$. All elements of the three search points are assumed to be discrete.

(M-STEP2) $\boldsymbol{D}^{k}$ is determined according to Eq. (11). An illustrative example of how to produce $\boldsymbol{D}^{k}$ is shown in Fig. 3.

In order to determine the $i$-th element of $\boldsymbol{D}^{k}$, a random number $r$ between $[0,1]$ is generated. If $r$ is less than $F, D_{i}^{k}$ inherits from $x_{r 3, i}{ }^{k}$. Otherwise, $D_{i}^{k}$ inherits from $x_{r 2, i}{ }^{k}$. (M-STEP3) $v_{d}^{k}$ is determined. In order to determine the $i$-th element of $\boldsymbol{v}_{d}{ }^{k}$, a random number $r$ between [0,1] is generated. If $r$ is less than $0.5, v_{d, i}{ }^{k}$ inherits from $D_{i}^{k}$. Otherwise, $v_{d, i}{ }^{k}$ inherits from $x_{r 1},{ }^{k}$. An illustrative example of this step, which uses $\boldsymbol{D}^{k}$ produced in Fig. 3, is shown in Fig. 4. 
Fig. 3 An illustrative example of how to produce $D^{k}$.
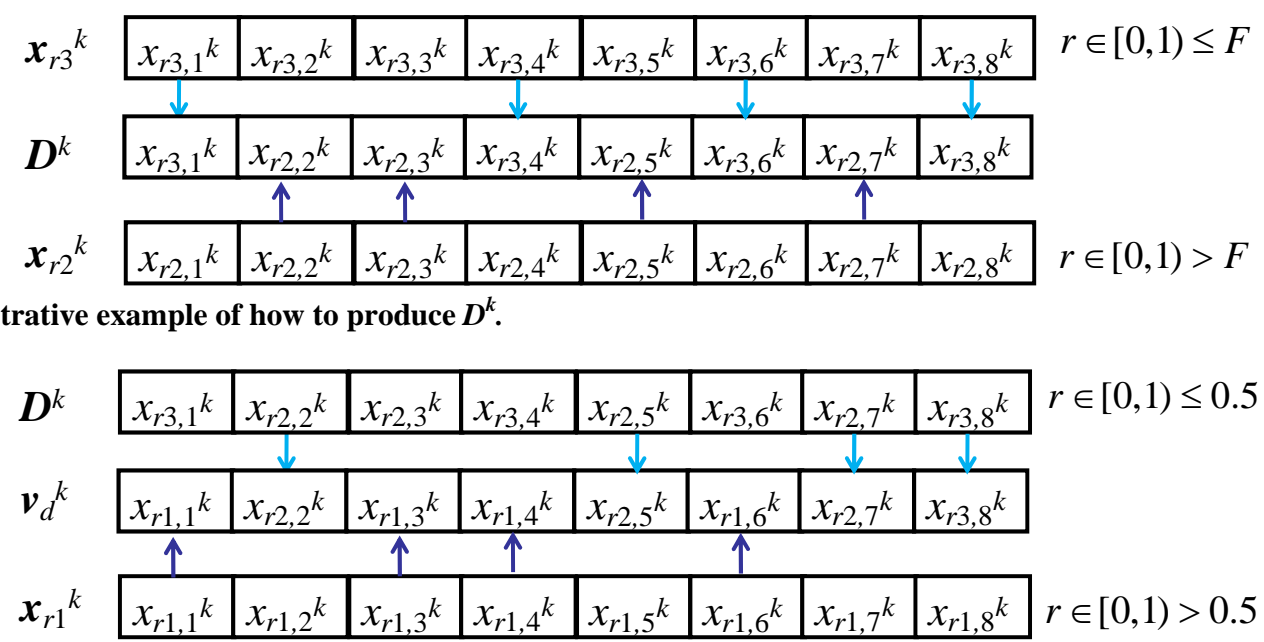

Fig. 4 An illustrative example of how to produce $v_{d}{ }^{k}$.

In order to produce a new search point $\boldsymbol{u}_{d}{ }^{k}$, the crossover between $\boldsymbol{x}_{d}{ }^{k}$ and $\boldsymbol{v}_{d}{ }^{k}$ is applied.

\subsection{Initialization of All Search Points}

In DE/1/rand/bin, three search points $\left(\boldsymbol{x}_{r 1}{ }^{k}, \boldsymbol{x}_{r 2}{ }^{k}\right.$, and $\left.\boldsymbol{x}_{r 3}{ }^{k}\right)$ are selected at random. It is expected that this random selection will enhance the global search. However, a new search point $\boldsymbol{u}_{d}{ }^{k}$ is obtained only when the objective function is improved. Therefore, simple application of Eqs. (11) and (14) will lead to a local minimum. In the proposed DDE, in order to escape from a local minimum, all search points are initialized. Thus, when the standard deviation of objective function is less than a threshold $\varepsilon$, all search points are randomly distributed in the design variable space. By initializing all search points, it is expected that diversity among search points will be maintained throughout the search process. As a result, it is expected that a global minimum can be found.

\subsection{Handling the Constraints}

In population-based optimization techniques, various penalty functions are often employed to handle behavior constraints [21]. In this paper, we construct the augmented objective function to be minimize according to the following penalty function [22]:

$$
\begin{gathered}
F(\boldsymbol{x})=f(\boldsymbol{x})+R \times \text { penalty } \rightarrow \min \\
R=(1+|f(\boldsymbol{x})|)^{q}
\end{gathered}
$$

$$
\text { penalty }=\left\{\begin{array}{cc}
\sum_{j=1} \exp \left(1+g_{j}(\boldsymbol{x})\right) & \cdots g_{j}(\boldsymbol{x})>0 \\
0 & \cdots g_{j}(\boldsymbol{x}) \leq 0
\end{array}\right.
$$

In Eq. (16) $q$ is a real number greater than 1. In this paper, $q$ is set to 2 . The penalty parameter $R$ in Eq. (16) is automatically determined using the penalty function approach presented above.

\subsection{Handling of Mixed Design Variables}

In the case of the mixed design variables, the components of the design variables can be expressed as follows:

$$
\boldsymbol{x}=\left(\boldsymbol{x}^{C}, \boldsymbol{x}^{D}\right)^{T}
$$

where $\boldsymbol{x}^{C}=\left(x_{1}, x_{2}, \cdots, x_{m}\right)^{T}$ and $\boldsymbol{x}^{D}=\left(x_{m+1}^{c}, x_{m+2}^{c}, \cdots, x_{m+n}^{c}\right)^{T}$ represent the components of continuous and discrete design variables, respectively. In this case, we have to pay attention to mutation. In mutation, Eqs. (11) and (14) are applied to the components of the discrete design variables, while Eq. (5) is applied to the components of the continuous design variables.

\subsection{Proposed DDE Algorithm}

The proposed DDE algorithm is shown in Fig. 5. In Fig. 5, STD represents the standard deviation of the objective function, and count denotes the number of convergence. The proposed DDE algorithm terminates with $k_{\max }$. 


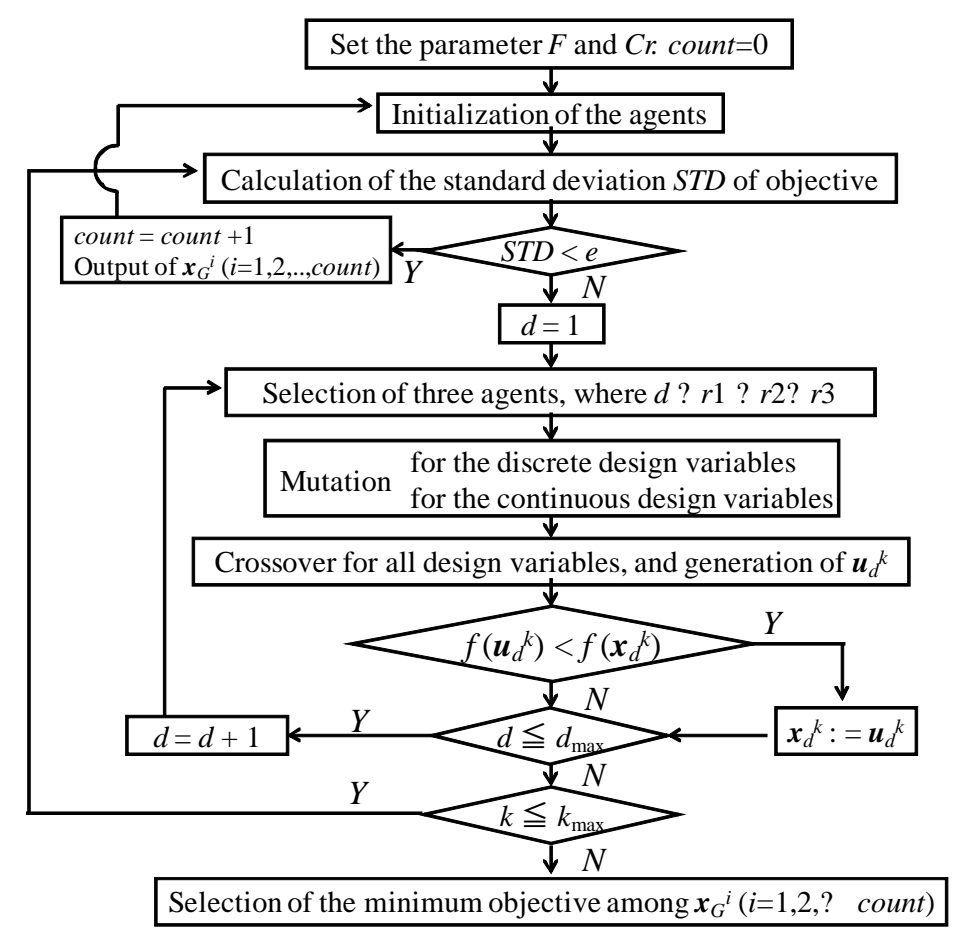

Fig. 5 The proposed DDE algorithm.

In comparison with the $\mathrm{DE} / 1 / \mathrm{rand} / \mathrm{bin}$ algorithm for continuous design variables, the main differences of the proposed DDE are summarized as follows:

All search points are randomly distributed on the discrete points.

If the standard deviation of the objective function is less than a threshold $\varepsilon$, then all search points are initialized.

\section{Numerical Examples}

Through typical benchmark problems, the validity of the proposed SDDE is examined. In all numerical examples, the mutation ratio $F$ is set to 0.6 , and the crossover ratio $\mathrm{Cr}$ is set to 0.5 . The threshold $\varepsilon$ is set to $1.0 \times 10^{-5}$. For each benchmark problem, fifty trials are performed with different random seeds.

\subsection{Optimum Design of A Gear Train}

Fig. 6 shows a simplified configuration of a compound gear train that has been investigated by many researchers [23-28]. It is desired to produce a gear ratio, or velocity ratio, as close as possible to 1/6.931. The gear ratio for the gear train is:

$$
\frac{T_{d} T_{b}}{T_{a} T_{f}}
$$

where $T_{a}, T_{b}, T_{d}$, and $T_{f}$ are the number of teeth on gears $A, B, D$, and $F$, respectively. These numbers are considered as the design variables. For each gear, the number of teeth is an integer value between 12 and 60 . Thus, the mathematical formulation of this problem is defined as follows:

$$
\begin{gathered}
f(x)=\left(\frac{1}{6.931}-\frac{x_{1} x_{2}}{x_{3} x_{4}}\right)^{2} \rightarrow \min \\
12 \leq x_{i} \leq 60 \quad i=1,2,3,4
\end{gathered}
$$

The number of search points is set to 30 , and the maximum number of search iterations is set to 200. The results are listed in Table 1. Typical histories of the objective function and the standard deviation of the objective function are shown in Fig. 7, in which the dashed line denotes the history of the objective function and the solid line denotes the history of the standard deviation of the objective function. We obtained the global minimum in all trials. As the result, the standard deviation of the objective function is zero.

It is clear from Fig. 7 that the global minimum can be found in approximately 50 iterations (1500 function 
evaluations). In addition, the initialization of all search points results in escaping from the local minima. Therefore, a global minimum cannot be found without initializing all search points. It is well known that this problem has four global minima, and in our experiment, we obtained the global minima listed in Table 2. The objective function at the global minima obtains the values listed in Table 2.

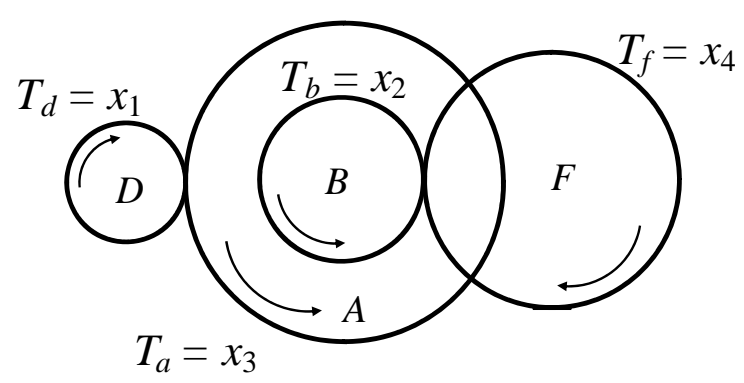

Fig. 6 Compound gear train.

Table 1 Comparison of results for the gear train problem.

\begin{tabular}{llllllll}
\hline & $\begin{array}{l}\text { Sandgren } \\
(23)\end{array}$ & $\begin{array}{l}\text { Loh } \\
(24)\end{array}$ & $\begin{array}{l}\text { Zhang } \\
(25)\end{array}$ & $\begin{array}{l}\text { Wu } \\
(26)\end{array}$ & $\begin{array}{l}\text { Lin } \\
(27)\end{array}$ & $\begin{array}{l}\text { Guo } \\
(28)\end{array}$ & $\begin{array}{l}\text { Proposed } \\
\text { SDDE }\end{array}$ \\
\hline x1 & 18 & 19 & 30 & 19 & 19 & 16 & 16 \\
x2 & 22 & 16 & 15 & 16 & 16 & 19 & 19 \\
x3 & 45 & 42 & 52 & 43 & 49 & 43 & 43 \\
x4 & 60 & 50 & 60 & 49 & 43 & 49 & 49 \\
& 5.70 & 2.33 & 2.36 & 2.70 & 2.70 & 2.70 & 2.7 \\
obj. & E-06 & E-07 & E-09 & E-12 & E-12 & E-12 & E-12 \\
\hline
\end{tabular}

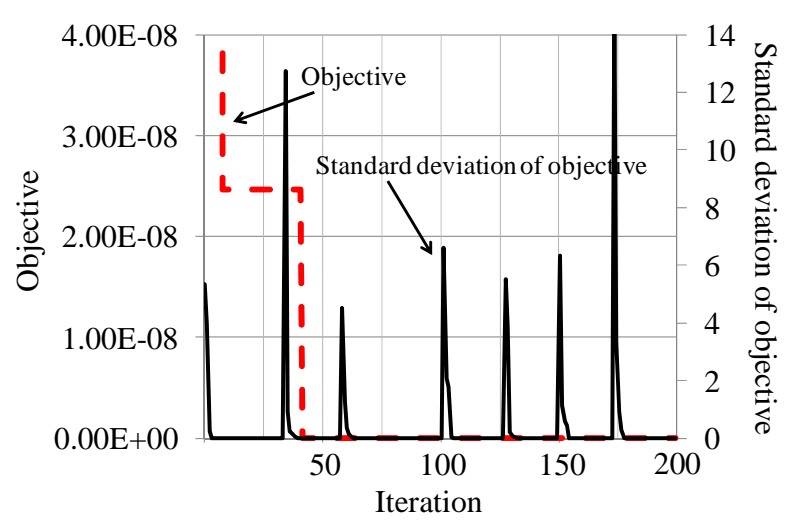

Fig. 7 Histories of the objective function and the standard deviation of the objective function.

Table 2 Other global minima of the gear train problem.

\begin{tabular}{llll}
\hline $\mathrm{x}_{1}$ & $\mathrm{x}_{2}$ & $\mathrm{x}_{3}$ & $\mathrm{x}_{4}$ \\
\hline 19 & 16 & 43 & 49 \\
19 & 16 & 49 & 43 \\
16 & 19 & 49 & 43 \\
\hline
\end{tabular}

\subsection{Optimum Design of A Truss Structure}

Let us consider the minimum weight design of a three bar truss, as shown in Fig. 8 [10, 13, 29]. Vertical and horizontal loads $P$ are applied at a node. The structural weight is minimized under the constraint that the stress in all bars should be smaller than the allowable stress $\sigma_{\max }$. Here we introduce $x_{i}=A_{\uparrow} \sigma_{\max } / P$. The mathematical formulation of the problem is as follows:

$$
\begin{gathered}
f(x)=2 x_{1}+x_{2}+\sqrt{2} x_{3} \rightarrow \min \\
g_{1}(x)=\frac{\sqrt{3} x_{2}+1.932 x_{3}}{1.5 x_{1} x_{2}+\sqrt{2} x_{2} x_{3}+1.319 x_{1} x_{3}}-1 \leq 0 \\
g_{2}(x)=\frac{0.634 x_{1}+2.828 x_{3}}{1.5 x_{1} x_{2}+\sqrt{2} x_{2} x_{3}+1.319 x_{1} x_{3}}-1 \leq 0 \\
g_{3}(x)=\frac{0.5 x_{1}-2 x_{2}}{1.5 x_{1} x_{2}+\sqrt{2} x_{2} x_{3}+1.319 x_{1} x_{3}}-1 \leq 0 \\
g_{4}(x)=-\frac{0.5 x_{1}-2 x_{2}}{1.5 x_{1} x_{2}+\sqrt{2} x_{2} x_{3}+1.319 x_{1} x_{3}}-1 \leq 0 \\
x \in\{0.1,0.2,0.3,0.5,0.8,1.0,1.2\}
\end{gathered}
$$

The number of search points is set to 10 , and the maximum number of iterations is set to 20 . Thus, the maximum number of function evaluations is 200 . The global minimum is $x_{\mathrm{G}}=(1.2,0.5,0.1)^{\mathrm{T}}$, and the objective function at the global minimum is $\mathrm{f}\left(x_{\mathrm{G}}\right)=3.0414$. Using the proposed algorithm with different random seeds, we always found the global minimum through all trials. Conversely, $\mathrm{Li}$ and Chou [29] occasionally failed to find the global minimum. This problem was previously solved using the penalty function approach by Shin et al. [13]. In Ref. [13], various penalty coefficients of the penalty function were adjusted, and many local minima were obtained. The results by Shin et al. indicate that in the penalty function approach, it is difficult to adjust the penalty coefficient. The proposed DDE requires no additional parameters for the discrete design variables, and can handle discrete design variables in a simple manner.

\subsection{Optimum Design of Pressure Vessel}

One of the most famous MDNLPs is the optimum design of a pressure vessel, as shown in Fig. 9. 


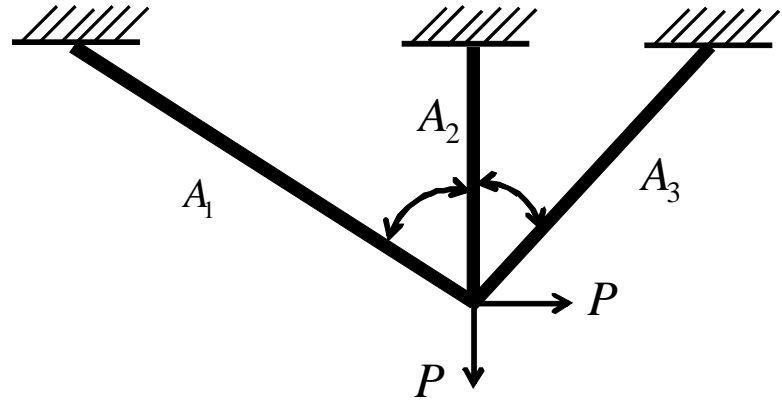

Fig. 8 Minimum weight design of a three bar truss.

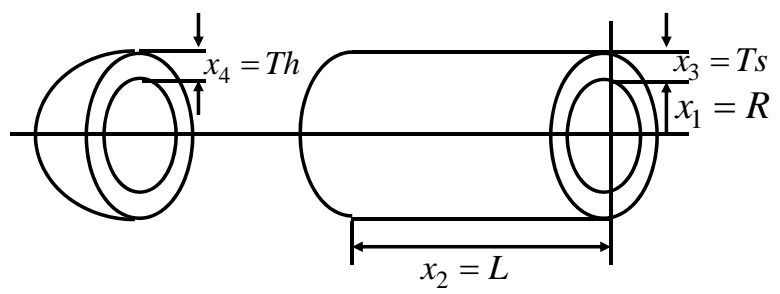

Fig. 9 Optimum design of a pressure vessel.

The design variables are radius $R=x_{1}$, length $L=x_{2}$, thickness $T s=x_{3}$, and thickness $T h=x_{4}$. The cost is minimized subject to four constraints. Though several formulations have been reported, the optimum design is formulated according to Ref. [30], as follows:

$$
\begin{gathered}
f(x)=0.6224 x_{1} x_{2} x_{3}+1.7781 x_{1}^{2} x_{4} \\
+3.1661 x_{2} x_{3}^{2}+19.84 x_{1} x_{3}^{2} \rightarrow \min \\
g_{1}(x)=\frac{0.0193 x_{1}}{x_{3}}-1 \leq 0 \\
g_{2}(x)=\frac{0.00954 x_{1}}{x_{4}}-1 \leq 0 \\
g_{3}(x)=\frac{x_{2}}{240}-1 \leq 0 \\
g_{4}(x)=\frac{1296000-\frac{4}{3} \pi x_{1}^{3}}{\pi x_{1}^{2} x_{2}}-1 \leq 0
\end{gathered}
$$

$$
\begin{gathered}
10 \leq x_{1}, x_{2} \leq 200 \\
0.0625 \leq x_{3}, x_{4} \leq 6.1875
\end{gathered}
$$

where $x_{3}$ and $x_{4}$ are the discrete design variables, which are the integer multiples of 0.0625. $x_{1}$ and $x_{2}$ are the continuous design variables. The number of search points is set to 50 , and the maximum number of search iterations is set to 1000 . The best results obtained with the proposed DDE are listed in Table 3. In addition, the histories of the objective and the standard deviation of the objective function are shown in Fig. 10, in which the dashed line denotes the history of the objective function and the solid line denotes the history of the standard deviation of the objective function.

Although the proposed DDE could not obtain the global minimum, it obtained a quasi-optimum solution. The initialization of all search points is introduced for the global search. If all search points are not initialized,

\begin{tabular}{|c|c|c|c|c|c|c|c|c|}
\hline & Sandgren $^{(23)}$ & Zhang $^{(25)}$ & $\mathrm{Wu}^{(26)}$ & $\operatorname{Lin}^{(27)}$ & $\mathrm{Guo}^{(28)}$ & Proposed Method & $\mathrm{He}^{(30)}$ & Kitayama $^{(12)}$ \\
\hline $\mathrm{x}_{1}(\mathrm{R}$ [inch]) & & N/A & 58.1978 & N/A & 58.2900 & 45.3368 & 42.0980 & 42.3710 \\
\hline $\mathrm{x}_{2}(\mathrm{~L}[\mathrm{inch}])$ & 117.7010 & N/A & 44.2930 & N/A & 43.7000 & 140.2539 & 176.6360 & 173.4170 \\
\hline $\mathrm{x}_{3}$ (Ts [inch]) & 1.1250 & N/A & 1.1250 & N/A & 1.1250 & 0.8750 & 0.8125 & 0.8125 \\
\hline $\mathrm{x}_{4}($ Th [inch]) & 0.6250 & N/A & 0.6250 & N/A & 0.6250 & 0.5000 & 0.4375 & 0.4375 \\
\hline$g_{1}(x)$ & -1.0000 & N/A & -0.0016 & N/A & 0.0000 & 0.0000 & 0.0000 & 0.0000 \\
\hline $\mathrm{g}_{2}(\mathrm{x})$ & -1.0000 & N/A & -0.1117 & N/A & -0.0689 & -0.1350 & -0.0820 & -0.0760 \\
\hline$g_{3}(x)$ & -0.5096 & N/A & -0.8154 & N/A & -0.8179 & -0.4156 & -0.2640 & -0.2770 \\
\hline $\mathrm{g}_{4}(\mathrm{x})$ & \#DIV/0! & N/A & -0.0021 & N/A & -0.0001 & 0.0000 & 0.000 & 0.0000 \\
\hline Objective [\$] & 8129.8000 & 7197.9000 & 7207.7748 & 7197.7000 & 7198.3058 & 6318.9492 & 6059.7143 & 6029.8740 \\
\hline
\end{tabular}
the search terminates at the local minimum found by Sandgren [23]. The initialization of all search points led to finding a quasi-optimum solution.

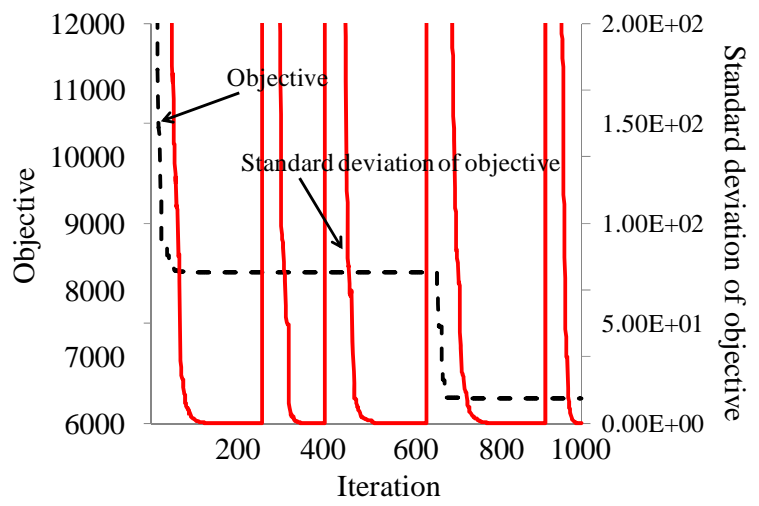

Fig. 10 Histories of the objective function and the standard deviation of the objective function.

Table 3 Comparison of results for the pressure vessel problem. 


\subsection{Other Benchmark Test Problems}

The benchmark problems considered from literature are listed in Table 4, and they were solved by the proposed DDE. In all benchmark problems, the number of search points was set to 10 , and the maximum number of search iterations was set to 20. Thus, the maximum number of function evaluations was 200. Fifty trials were performed. In all benchmark problems, the proposed DDE always found the global minimum in less than 20 function evaluations.

Table 4 Benchmark problems of the discrete or mixed design variable problem.

\begin{tabular}{|c|c|c|c|c|}
\hline No. & Ref. & Formulation & Global minimum & Objective \\
\hline 1 & 31 & $\begin{array}{l}f(x)=7 x_{1}^{2}+6 x_{2}^{2}+8 x_{3}^{2}-6 x_{1} x_{2}+4 x_{2} x_{3} \\
\quad-15.8 x_{1}-93.2 x_{2}-63 x_{3}+500 \rightarrow \min \\
g_{1}(x)=142 x_{1}+172 x_{2}+118 x_{3}-1992 \leq 0 \\
g_{2}(x)=98 x_{1}+114 x_{2}+44 x_{3}-1162 \leq 0 \\
g_{3}(x)=40 x_{1}+72 x_{2}+34 x_{3}-703 \leq 0 \\
0 \leq x_{1}, x_{2}, x_{3} \leq 10 \quad x_{1}, x_{2}, x_{3}: \text { int }\end{array}$ & $\boldsymbol{x}_{G}=(2,7,3)^{T}$ & $f\left(x_{G}\right)=69$ \\
\hline 2 & 31 & $\begin{array}{l}f(x)=\left(x_{1}-8\right)^{2}+\left(x_{2}-2\right)^{2} \rightarrow \min \\
g_{1}(x)=0.1 x_{1}^{2}-x_{2} \leq 0 \\
g_{2}(x)=\frac{x_{1}}{3}+x_{2}-4.5 \leq 0 \\
0 \leq x_{1}, x_{2} \leq 6 \quad x_{1}, x_{2}: \text { int }\end{array}$ & $x_{G}=(4,2)^{T}$ & $f\left(x_{G}\right)=16$ \\
\hline 3 & 31 & $\begin{array}{l}f(x)=-x_{1}-1.8 x_{2} \rightarrow \min \\
g_{1}(x)=x_{1}^{2}+\left(x_{2}+6\right)^{2}-85 \leq 0 \\
g_{2}(x)=1-x_{1}^{2} \leq 0 \\
0 \leq x_{1} \leq 8 \quad 0 \leq x_{2} \leq 4 \quad x_{1}, x_{2}: \text { int }\end{array}$ & $x_{G}=(6,1)^{T}$ & $f\left(x_{G}\right)=-7.8$ \\
\hline 4 & 32 & $\begin{array}{l}f(x)=100\left(x_{2}-x_{1}^{2}\right)^{2}+\left(1-x_{1}\right)^{2} \rightarrow \text { min } \\
-0.55 \leq x_{1}, x_{2} \leq 4.95 \quad \text { interval: } 0.55\end{array}$ & $\boldsymbol{x}_{G}=(1.65,2.75)^{T}$ & $f\left(x_{G}\right)=0.498125$ \\
\hline 5 & 32 & $\begin{array}{l}f(x)=0.1\left[12+x_{1}^{2}+\frac{1+x_{2}^{2}}{x_{1}^{2}}+\frac{x_{1}^{2} x_{2}^{2}+100}{\left(x_{1} x_{2}\right)^{4}}\right] \rightarrow \min \\
-5 \leq x_{1}, x_{2} \leq 5 \quad x_{1}, x_{2}: \text { int }\end{array}$ & $\begin{array}{l}\boldsymbol{x}_{G}=(2,2)^{T} \\
\boldsymbol{x}_{G}=(-2,2)^{T} \\
\boldsymbol{x}_{G}=(-2,-2)^{T} \\
\boldsymbol{x}_{G}=(2,-2)^{T}\end{array}$ & $f\left(x_{G}\right)=1.7703125$ \\
\hline 6 & 24 & $\begin{array}{l}f(x)=-9 x_{1}^{2}+10 x_{1} x_{2}-50 x_{1}+8 x_{2}+460 \rightarrow \min \\
g_{1}(x)=x_{1}-\left(0.2768 x_{2}^{2}-0.235 x_{2}+3.718\right) \leq 0 \\
g_{2}(x)=x_{1}+0.019 x_{2}^{3}-0.446 x_{2}^{2} \\
\quad+3.98 x_{2}-15.854 \leq 0 \\
0 \leq x_{1}, x_{2} \leq 10 \quad x_{1}, x_{2}: \text { int }\end{array}$ & $\boldsymbol{x}_{G}=(5,3)^{T}$ & $f\left(x_{G}\right)=159$ \\
\hline 7 & 33 & $\begin{array}{l}f(x)=x_{1}^{2}+x_{2}^{2} \rightarrow \min \\
g_{1}(x)=1 / x_{1}+1 / x_{2}-2 \leq 0 \\
x_{1} \in\{0.3,0.7,0.8,1.2,1.5,1.8\} \\
x_{2} \in\{0.4,0.8,1.1,1.4,1.6\}\end{array}$ & $x_{G}=(0.8,1.4)^{T}$ & $f\left(x_{G}\right)=2.6$ \\
\hline
\end{tabular}




\section{Conclusions}

In this paper, we proposed a differential evolution algorithm for discrete design variables. The proposed DDE is based on the DE/1/rand/bin algorithm used for continuous design variables. The proposed DDE can directly handle discrete design variables, and the mutation ratio is regarded as the exchange probability. In order to maintain diversity throughout the search process, all search points were initialized. By initializing all search points, the global or quasi-optimum solution could be obtained. In the proposed DDE, no modifications are required to handle the discrete design variables. The validity of the proposed DDE was verified through typical numerical examples.

\section{References}

[1] R. Storn and K. V. Price, Differential evolution - A simple and efficient heuristic for global optimization over continuous spaces, J. of Global Optimization 11 (1997) 341-359.

[2] K. V. Price, R. Storn and J. A. Lampinen, Differential Evolution: A Practical Approach to Global Optimization, Springer, 2006.

[3] V. Feoktistov, Differential Evolution: In Search of Solutions, Springer, 2006.

[4] U. K. Chakraborty (Ed.), Advances in Differential Evolution, Springer, 2008.

[5] A. Qing, Differential Evolution: Fundamentals and Applications in Electrical Engineering, John Wiley \& Sons, 2009.

[6] D. Zaharie, Influence of crossover on the behavior of differential evolution algorithms, Applied Soft Computing 9 (2009) 1126-1138.

[7] J. Tvrdik, Adaptation in differential evolution: A numerical comparison, Applied Soft Computing 9 (2009) 1149-1155.

[8] H. Tang, S. Xue and C. Fan, Differential evolution strategy for structural system identification, Computers and Structures 86 (2008) 2004-2012.

[9] G. Venter and J. S. Sobieski, Multidisciplinary optimization of a transport aircraft wing using particle swarm optimization, Struct. and Multidisc. Optim. 26 (1-2) (2004) 121-131.

[10] S. S. Rao, Engineering Optimization: Theory and Practice, Wiley InterScience, 1996.
[11] J. S. Arora and M. W. Huang, Methods for optimization of nonlinear problems with discrete variables: A review, Struct. Optim. 8 (1994) 69-85.

[12] S. Kitayama, M. Arakawa and K. Yamazaki, Penalty function approach for the mixed discrete non-linear problems by particle swarm optimization, Structural and Multidisciplinary Optimization 32 (3) (2006) 191-202.

[13] D. K. Shin, Z. Gurdal and O. H. Griffin Jr, A penalty approach for nonlinear optimization with discrete design variables, Engineering Optimization 16 (1990) 29-42.

[14] G. C. Onwubolu and D. Davendra (Eds.), Differential Evolution: A Handbook for Global Permutation-Based Combinatorial Optimization, Springer, 2009.

[15] B. Qian, L. Wang, R. Hu, D. X. Huang and X. Wang, A DE-based approach to no-wait flow-shop scheduling, Computers and Industrial Engineering 57 (2009) 787-805.

[16] Q. K. Pan, M. F. Tasgetiren and Y. C. Liang, A Discrete differential evolution algorithm for the permutation flowshop scheduling problem, Computers and Industrial Engineering 55 (2008) 795-816.

[17] W. X. Xia and Z. M. Wu, A hybrid particle swarm optimization approach for the job-shop scheduling problem, International Journal of Advanced Manufacturing Technology 29 (2006) 360-366.

[18] Q. K. Pan and L. Wang, No-idle permutation flow shop scheduling based on a hybrid discrete particle swarm optimization algorithm, International Journal of Advanced Manufacturing Technology 39 (2008) 796-807.

[19] Q. K. Pan, L. Wang, M. F. Tasgetiren and B. H. Zhao, A hybrid discrete particle swarm optimization algorithm for the no-wait flow shop scheduling problem with makespan criterion, International Journal of Advanced Manufacturing Technology 38 (2008) 337-347.

[20] A. P. Engelbrecht, Fundamentals of Computational Swarm Intelligence, Wiley, 2005.

[21] K. Deb, Multi-Objective Optimization Using Evolutionary Algorithms, Wiley, 2001.

[22] S. Kitayama, K. Yamazaki and M. Arakawa, Adaptive range particle swarm optimization, Optimization and Engineering 10 (4) (2009) 575-597.

[23] E. Sandgren, Nonliear integer and discrete programming in mechanical design optimization, ASME/J. of Mechanical Design 112 (1990) 223-229.

[24] H. T. Loh and P. Y. Papalambros, Computational implementation and tests of a sequential linearization algorithm for mixed-discrete nonlinear design optimization, ASME/J. of Mechanical Design 113 (1991) 335-345. 
[25] C. Zhang and H. P. Wang, Mixed-discrete nonlinear optimization with simulated annealing, Engineering Optimization 21 (1993) 277-291.

[26] S. J. Wu and P. T. Chow, Genetic algorithms for nonlinear mixed discrete-integer optimization problems via meta-genetic parameter optimization, Engineering Optimization 24 (1995) 137-159.

[27] S. S. Lin, C. Zhang and H. P. Wang, On mixed-discrete nonlinear optimization problems: A comparative study, Engineering Optimization 23 (1995) 287-300.

[28] C. X. Guo, J. S. Hu, B. Ye and Y. J. Cao, Swarm intelligence for mixed-variable design optimization, Journal of Zhejiang University Science 5 (7) (2004) 851-860.

[29] H. L. Li and C. T. Chou, A global approach for nonlinear mixed discrete programming in design optimization, Engineering Optimization 22 (1994) 109-122.

[30] S. He, E. Prempain and Q. H. Wu, An improved particle swarm optimizer for mechanical design optimization problems, Engineering Optimization 36 (5) (2004) 585-605.

[31] H. T. Loh and P. Y. Papalambros, A sequential linearization approach for solving mixed-discrete nonlinear design optimization problems, ASME/J. of Mechanical Design 113 (1991) 325-334.

[32] J. F. Fu, R. G. Fenton and W. Cleghorn, A Mixed Integer-discrete-continuous programming method and its application to engineering design optimization, Engineering Optimization 17 (1991) 263-280.

[33] G. R. Olsen, Method for nonlinear optimization with discrete design variables, AIAA J. 27 (11) (1989) 1584-1589.

[34] C. A. Floudas (Ed), Handbook of Test Problems in Local and Global Optimization, Kluwer Academic Publishers, Netherlands, 1999.

\section{Appendix}

The mutation and the crossover operations play an important role in the proposed DDE. For a better understanding, fortran 77 code of the mutation and crossover operations is presented as below:

$\mathrm{x} 0$ : random seed

r: random number between 0 and 1

agent: the number of search points

mrate0: mutation ratio

continuous: the number of continuous design variables

lower(i): the lower bound of the $i$-th design variable upper(i): the upper bound of the $i$-th design variable $\mathrm{xx}(\mathrm{i}, \mathrm{j}): j$-th design variable of the $i$-th search point ndv: the number of design variables ncon: the number of constraints

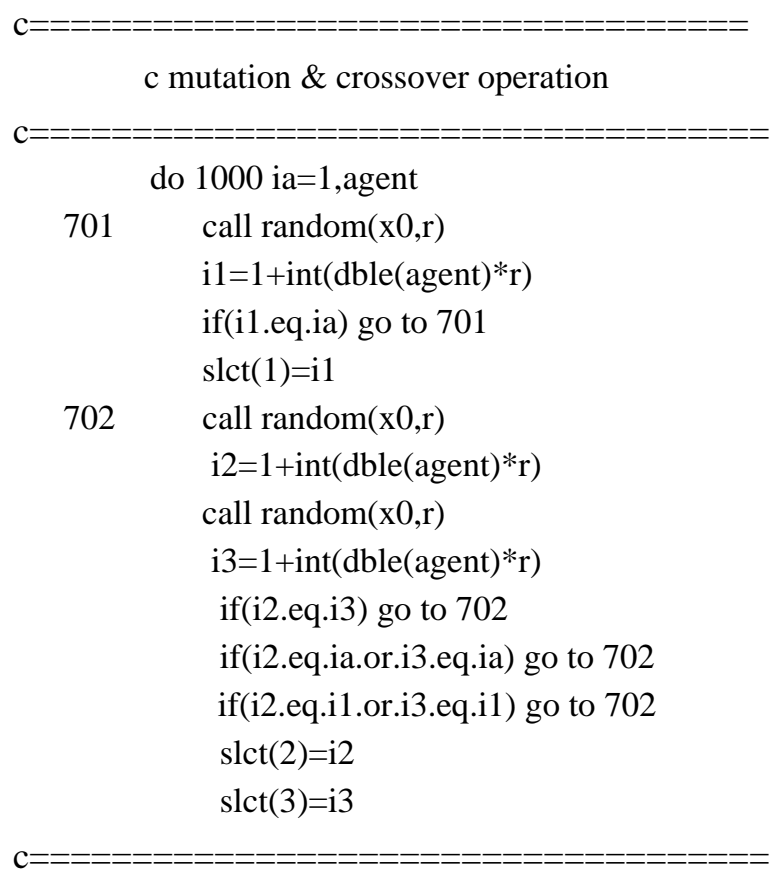

c mutation operation for discrete design variables

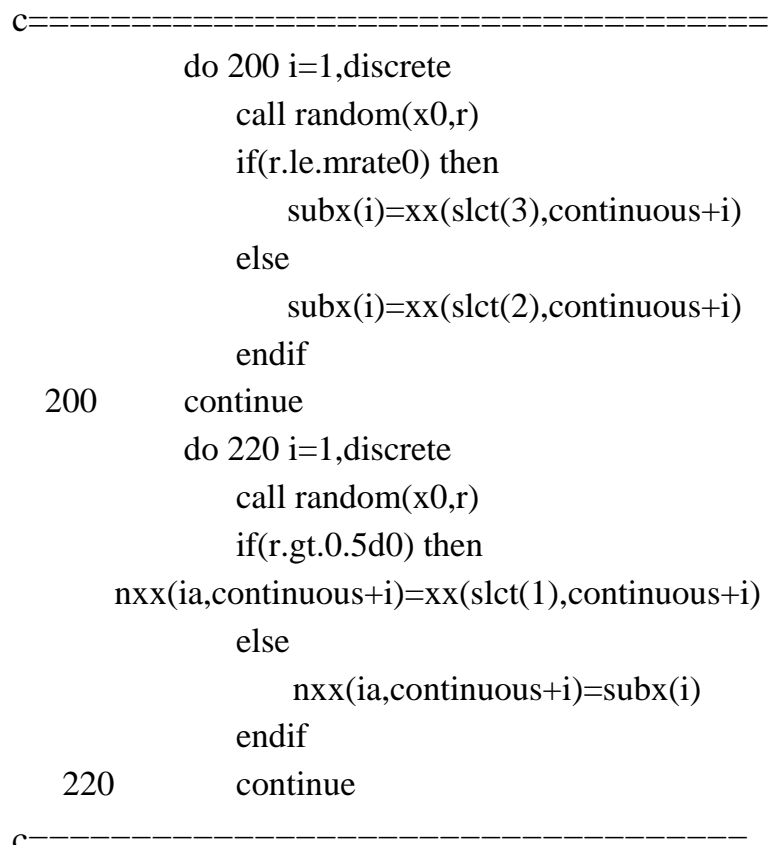

c mutation for continuous design variables

do $230 \mathrm{i}=1$,continuous

call random $(\mathrm{x} 0, \mathrm{r})$

$\operatorname{nxx}(\mathrm{ia}, \mathrm{i})=$ 


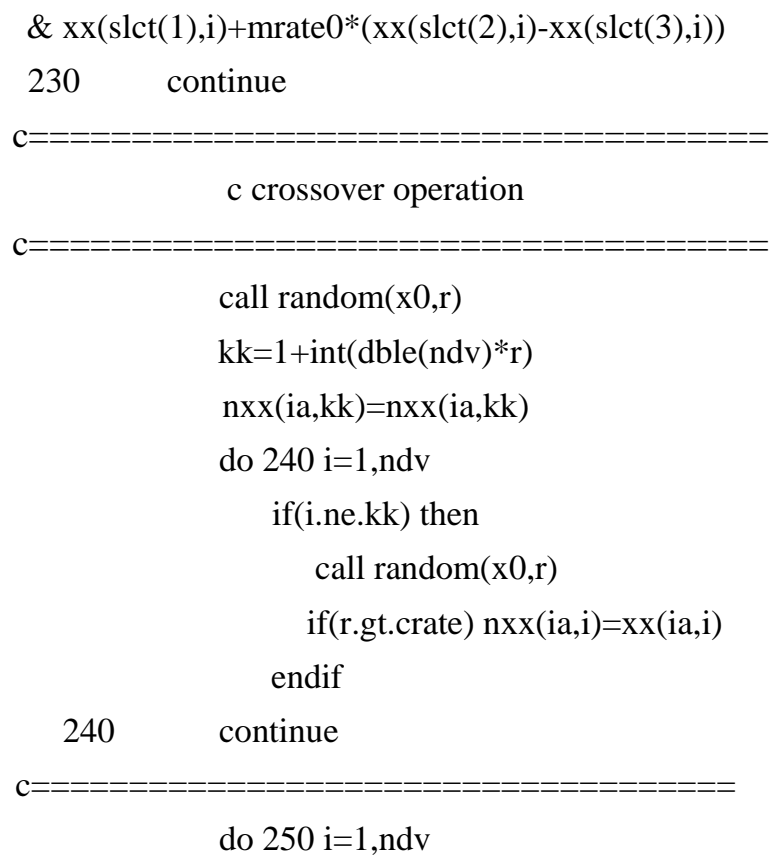

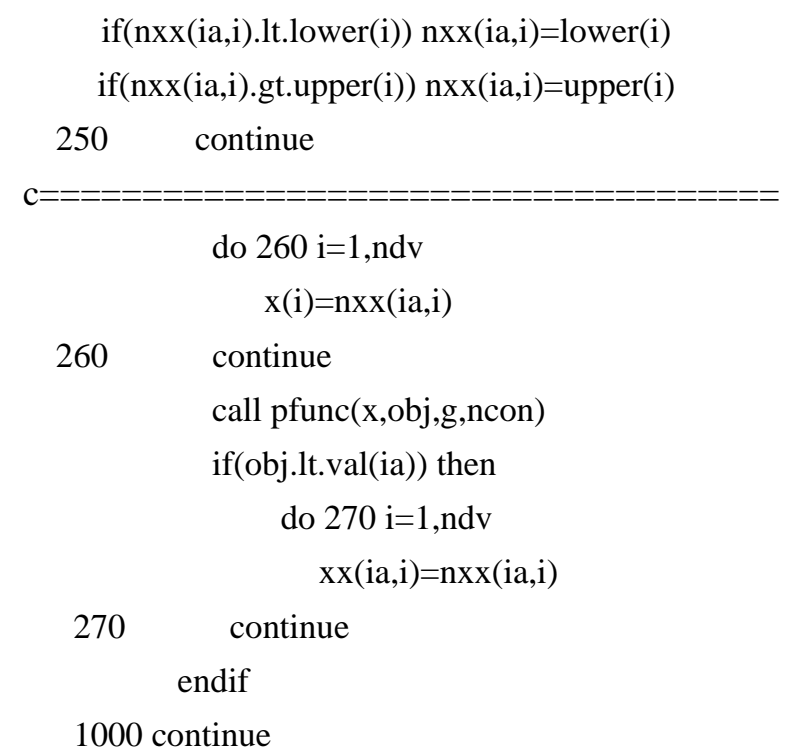

